$63^{\text {ème }}$ Congrès de la SFCO, 02023 (2015)

DOI:10.1051/sfco/20156302023

(C) Owned by the authors, published by EDP Sciences, 2015

\title{
COMMUNICATION
}

\section{Cemento-osseous dysplasia of the jawbones : systematic algorithm for diagnosis derived from retrospective data over a 5 -year period}

\author{
Maccotta M, Lescaille G, Baaroun V, Razouk O, Poulain Ch, Lachoubi M, Nguyen A, \\ Goudot P, Descroix V, Mauprivez C \\ Conf sfco, Groupe Hospitalier Pitié Salpétrière, 47-83 boulevard de l'Hopital, 75651 Paris
}

Cemento-osseous dysplasia (COD) are usually found in black women 40-50 years old. In 2005, the World Health Organization subdivided CODs into periapical, florid and other CODs (1).

The diagnosis of COD which can include several different but related entities, should consider tooth pulp vitality, the stage of lesion development and the possible co-existence of COD with other entities, namely simple bone cyst and osteomyelitis. Consequently, the diagnostic confusion often associated with COD has led to misinterpretation and mismanagement, often rendering these cases problematic $(2,3)$.

We have retrospectively examined panoramic radiograhs acquired in oral surgery department of the Pitié Salpetrière over 5-year period (31 december 2009 to 31 december 2014). We loocked for radiological findings that showed the typical appearance of periapical COD or florid COD.

Inclusion criteria were divided into four categories of radiographic features : 1/ localisation (mandible only, posterior teeth only, unilateral, solitary), 2/ periphery and shape (well-defined, round or oval, corticated border, radioloucent border, no peripheral sclerosis), 3/ Internal structure (radioluscent, mixed radiolucent-radiopaque, radiopaque, dense, cementum-like radiopacities), 4/ effect on surrounding structures (no endosteal and/or interradicular scalloping, intact lamina dura, normal periodontal ligament space, no root resorption, no hypercementosis, no cortical expansion, no displacement of anatomic structures, no periosteal reaction).

We identified 59 cases of COD and 16 cases were classified as possibly having a COD but did not fullfil enough critera to confirm the diagnosis. In addition to the radiopanoramic $\mathrm{X}$ rays, 8 patients underwent alveolar retro, 21 patients with a CT-scan or Cone Beam-CT, and 35 of a biopsy and / or sequestrectomy. While individual radiologic or histopathologic features are not specific to CO, they are pathognomonic when they occur in adult in the absence of other developmental abnormalities.

A diagnostic algorithm for bony lesions intially suspected to be COD will be presented during the session. In the early immature osteolytic stage, COD lesions may be confused with periapical lesion (i.e. radicular abscess, granuloma or cyst), particularly the small, solitary lesions.

This is an Open Access article distributed under the terms of the Creative Commons Attribution License 4.0, which permits unrestricted use, distribution, and reproduction in any medium, provided the original work is properly cited. 
As COD lesions mature, the presence of radiopacification can be confused with ossifiant fibroma fibrous dysplasia, cementoblastoma or osteoblastoma. In cases of florid COD, it's essential to exclude differential diagnoses such, cementoma gigantiform, Paget's disease, Gardner's syndrome, and chronic osteomyelitis,

Most COD cases are diagnosed on the basis of the radiographic features. Open exploration can be avoided in almost all cases by through radiological diagnosis, so surgical intervention is usually not necessary, as COD mentioned in his initial description. In atypical presentation, CT-scan or cone beam- CT is helpful to verify the no cortical expansion, no displacement of anatomic structures and no periosteal reaction. If the diagnosis is still not clear, it can confirm by biopsy.

(1) Barnes L, Eveson JW, Reichart P, Sidransky D, editors. Pathology and genetics of head and neck tumours. World Health OrganizationClassification of Tumours. Lyon: IARC Press; 2005.

(2) Galgano C, Samson J, Kuffer R, Lombardi T. Focal cemento-osseous dysplasia involving a mandibular lateral incisor. Int Endod J 2003; 36: 907-11.

(3) Horner K, Forman GH. Atypical simple bone cysts of the jaws. II: A possible association with benign fibro-osseous (cemental) lesions of the jaws. Clinical Radiology 1988; 39: 59-63.

Nom et adresse du conférencier

\section{Cédric MAUPRIVEZ}

Conf sfco Groupe Hospitalier Pitié Salpétrière

47-83 boulevard de l'Hopital

75651 Paris (France)

mauprivez.cedric@gmail.com 